\title{
Unraveling the cellular and molecular mechanisms of repetitive magnetic stimulation
}

\author{
Florian Müller-Dahlhaus ${ }^{1}$ and Andreas Vlachos ${ }^{2}$ * \\ ${ }^{1}$ Department of Neurology and Stroke, Hertie Institute for Clinical Brain Research, Eberhard-Karls-University Tübingen, Tübingen, Germany \\ ${ }_{2}^{2}$ Institute of Clinical Neuroanatomy, Neuroscience Center, Goethe-University Frankfurt, Frankfurt am Main, Germany
}

\section{Edited by:}

Jean Rossier, Ecole Superieure de Physique et de Chimie Industrielles de la Ville de Paris, France

\section{Reviewed by:}

Marc A. Maier, Université Paris Diderot, France

Xavier Moisset, INSERM U1107,

France

Daniel Ciampi De Andrade, Faculdade de Medicina da Universidade de São Paulo, Brazil

\section{*Correspondence:}

Andreas Vlachos, Institute of Clinical Neuroanatomy, Neuroscience Center, Goethe-University Frankfurt,

Theodor-Stern Kai 7, 60590 Frankfurt am Main, Germany

e-mail:a.vlachos@med.uni-frankfurt.de
Despite numerous clinical studies, which have investigated the therapeutic potential of repetitive transcranial magnetic stimulation (rTMS) in various brain diseases, our knowledge of the cellular and molecular mechanisms underlying rTMS-based therapies remains limited. Thus, a deeper understanding of rTMS-induced neural plasticity is required to optimize current treatment protocols. Studies in small animals or appropriate in vitro preparations (including models of brain diseases) provide highly useful experimental approaches in this context. State-of-the-art electrophysiological and live-cell imaging techniques that are well established in basic neuroscience can help answering some of the major questions in the field, such as (i) which neural structures are activated during TMS, (ii) how does rTMS induce Hebbian plasticity, and (iii) are other forms of plasticity (e.g., metaplasticity, structural plasticity) induced by rTMS? We argue that data gained from these studies will support the development of more effective and specific applications of rTMS in clinical practice.

Keywords: non-invasive brain stimulation, neurological diseases, neuropsychiatric disorders, Hebbian plasticity, metaplasticity, structural plasticity

\section{INTRODUCTION}

Galvani (1791), an Italian physician from Bologna, was among the first to demonstrate that electrical energy drives body function. He reported that the stimulation of the frog's sciatic nerve with a metal rod (charged with static electricity) caused contractions of the innervated muscle. Ever since this observation the question of how electrical signals in neural tissue translate into complex human behavior, both under physiological and pathological conditions, has attracted the interest of scientists and physicians around the world. Two centuries after Galvani's famous experiments, Barker and colleagues carried out a set of analogous experiments in human subjects. To evoke muscle contractions though, Barker etal. (1985) stimulated the brain, i.e., the motor cortex, of their subjects by employing a novel non-invasive stimulation technique termed transcranial magnetic stimulation (TMS). These experiments opened several new scientific avenues and provided a means to non-invasively assess cortical excitability and function in patients with brain diseases (for review see, Hallett, 2007).

Transcranial magnetic stimulation is based on the physical principle of electromagnetic induction: a strong electric current of up to several $\mathrm{kA}$ is discharged briefly $(<1 \mathrm{~ms}$ duration) through a TMS coil. This generates a short-lived, but strong electromagnetic field, which penetrates the scalp and skull and induces an electric field in the underlying brain tissue. By this mechanism TMS allows for the non-invasive activation of the cerebral cortex in awake and non-anesthetized human subjects (Figure 1A). More recently, experimental evidence has been provided that repetitive TMS (rTMS), i.e., trains of several hundred pulses can change the excitability of the human cortex for hours beyond the stimulation period (for review see, Ziemann et al., 2008). This observation has driven interest toward a therapeutic use of rTMS in neurological and neuropsychiatric disorders associated with alterations in cortical excitability (Ziemann, 2005; Edwards et al., 2008; George et al., 2013). However, the cellular and molecular mechanisms underlying rTMS-induced neural plasticity remain not well understood. Considering data, which demonstrate a substantial inter- and intra-individual variability of rTMS-induced after-effects (MüllerDahlhaus et al., 2008; Tecchio et al., 2008; Hamada et al., 2013), a better understanding of how TMS affects neural tissue is urgently needed.

Repetitive magnetic stimulation (rMS) of small animals and appropriate in vitro preparations represent suitable and highly useful experimental approaches in this context (Figures 1B,C). Several of these models have already been used successfully (e.g., Levkovitz et al., 1999; Meyer et al., 2009; Tokay et al., 2009; Benali et al., 2011; Gersner et al., 2011; Wang et al., 2011; Vlachos et al., 2012; Mix etal., 2013) and new important insights have been gained by these studies. Still, our knowledge of rMS-induced neural plasticity remains limited. The major focus of this perspective article is to discuss some of the open questions in the field and to illustrate how experimental approaches that are well established in basic neuroscience might help in addressing these questions. This attempt may provide a framework for future studies and could also attract the expertise of neuroscientists from other fields to join the endeavor of unraveling the cellular and molecular mechanisms of rTMS-induced neural plasticity. 


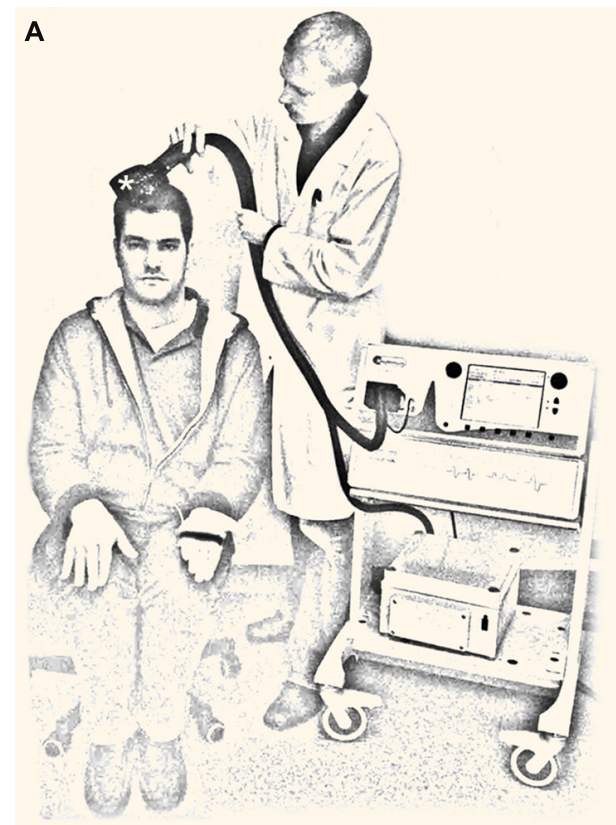

FIGURE 1 | (A) Non-invasive brain stimulation by transcranial magnetic stimulation (TMS). A single TMS pulse of sufficient intensity applied over the primary motor cortex hand area through a TMS coil (asterisk) induces a muscle contraction in the contralateral hand and elicits a motor-evoked potential in the target muscle. Repetitive TMS (rTMS; i.e., trains of several 100 pulses) can change cortical excitability for hours beyond the stimulation period, which has driven interest toward a therapeutic use of

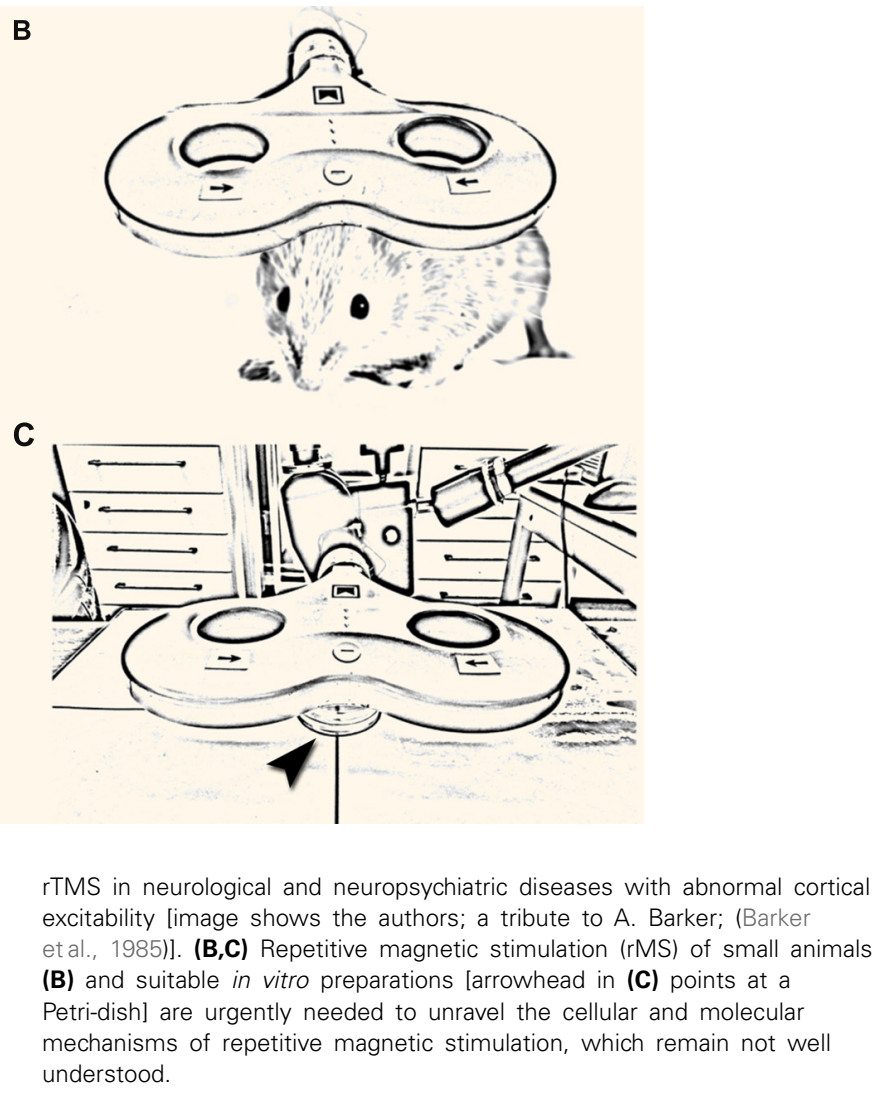

cells or even specific subcellular compartments depolarized by TMS?

\section{WHICH NEURAL STRUCTURES ARE ACTIVATED DURING TMS?}

While evidence has been provided that axons are the primary target of TMS (Figure 2A; Basser and Roth, 1991; Basser, 1994; Rotem and Moses, 2008), it is not known whether all axons of a particular orientation within the induced electric field will be depolarized. Considering the diverse functional and structural properties of neurons, differential effects on axons of inhibitory and excitatory neurons or even specific subtypes of a class of neurons are possible. In addition, TMS may depolarize certain axons at multiple locations or produce complex spike trains by activating recurrent networks (Edgley et al., 1997), even though single TMS pulses are applied. Likewise, the depolarization of specific axons may not only lead to the induction of anterograde propagating action potentials (aAPs), but will also produce backward propagating action potentials (bAPs), which can propagate into the dendritic tree and depolarize dendrites of a target neuron (Stuart and Sakmann, 1994). Thus, specific pre- and postsynaptic structures may be activated by TMS within the stimulated network (Figure 2A). Furthermore, direct or indirect effects on glial cells, mitochondria, intracellular calcium stores, and calcium buffers, polyribosomes, translation/transcription factors, specific molecular complexes such as adhesion molecules, ligand- or voltage-gated channels/receptors, 
A

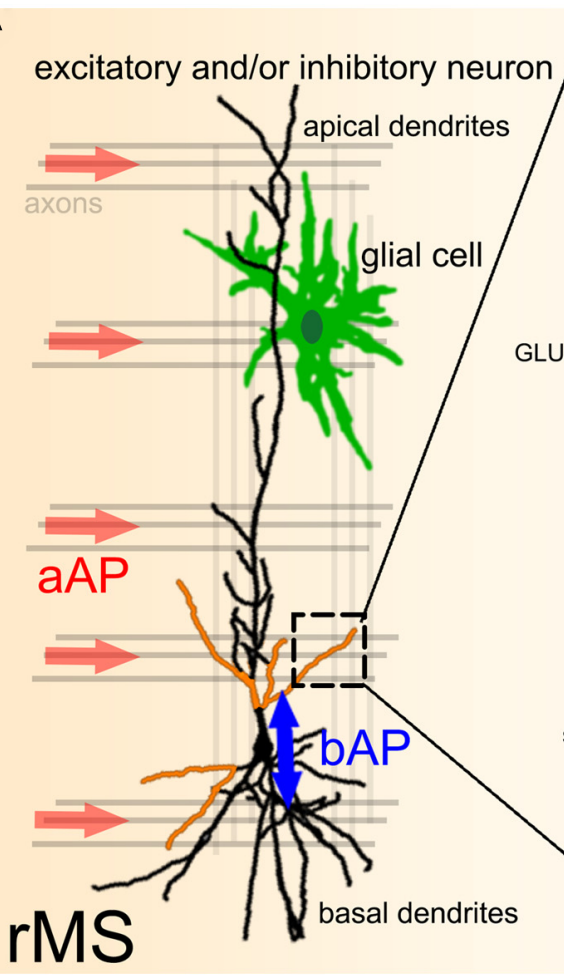

FIGURE 2 | Effects of repetitive TMS (rTMS) on single neurons within a network. (A) During TMS axons with a particular orientation within the induced electric field are depolarized. This leads to anterograde propagating action potentials (aAP). In addition, TMS may also initiate backward propagating APs (bAP) in the target neuron, or even directly depolarize specific dendritic segments (indicated by orange color). TMS-effects on other
B I| ligand-gated ion channels

II voltage-gated ion channels

metabotropic receptors

$X$ postsynaptic scaffolds

I adhesion molecules

polyribosomes neural structures, e.g., glial cells (astrocytes, microglia, oligodendrocytes) have not been investigated so far. The role of local circuits (e.g., recurrent networks, feed-forward, and feed-back inhibition) needs to be determined in this context as well. (B) Illustration of potential direct or indirect molecular targets of rTMS. For further details please see text [SA, spine apparatus organelle (Gray, 1959; Spacek, 1985)]. metabotropic receptors, postsynaptic scaffolds, and other cellular and molecular structures (Figure 2B) cannot be ruled out at present and thus warrant further investigation. Finally, the role of the stimulation parameters, e.g., size and geometry of the TMS coil, orientation of the coil relative to the stimulated tissue, distance from tissue, temporal dynamics of the electromagnetic field, and the stimulation protocol need to be considered (Chipchase et al., 2012). Hence, we are confronted with an enormous parameter space which can only be assessed systematically by acquiring functional data under highly controlled and standardized experimental conditions (ideally at the level of single identified cells).

Clearly, electrophysiological recordings of intact animals or suitable in vitro preparations represent the gold standard approach to determine the effects of TMS during stimulation. While local field potential recordings (e.g., single- or multi-electrode recordings) allow for the assessment of neural population activity (down to the level of single units), patch-clamp recordings (single- or multi-cell recordings, dual somato-dendritic recordings, recordings from astrocytes) are required to directly record TMS-induced changes in voltage, current, and firing rate of individual cells. However, these (and other suitable electrophysiological) experiments are not trivial to perform during stimulation due to the strong electromagnetic field that is generated by TMS.
An interesting solution to this problem is optical functional imaging (Scanziani and Hausser, 2009). Together with recent advances in microscopy it has become possible to visualize activity in neural tissue at high temporal and spatial resolution using proper chemical or biological sensors, even at the network level (Grewe et al., 2010). The development of novel indicators allows not only to image changes in membrane potential (Grinvald and Hildesheim, 2004; Perron etal., 2009; Marshel and Deisseroth, 2013) and monitor calcium transients (Grienberger and Konnerth, 2012; Akerboom et al., 2013; Chen et al., 2013), but to determine changes in intracellular chloride concentrations (Bregestovski etal., 2009; Berglund etal., 2011), cGMP levels (Bhargava et al., 2013), shifts in pH (Raimondo et al., 2012), and other functionally relevant molecules, both in vitro and in vivo (Smedemark-Margulies and Trapani, 2013). Furthermore, transgenic animals, (in utero) electroporation or transfection with viral vectors can be used to express genetically encoded biosensors (and/or other proteins) in selected cells, which allows for the assessment of specific cell types within neural networks (Packer et al., 2013). In addition, optogenetic approaches [e.g., the lightinduced depolarization or hyperpolarization of neural structures; (Tye and Deisseroth, 2012; see also Deisseroth and Schnitzer, 2013)] can be used to discern critical elements within the stimulated tissue during TMS. In combination with pharmacological 
and other genetic approaches these "contact-free" techniques represent state-of-the-art approaches to identify the neural structures that are activated during TMS, and to test for their contribution to rTMS-induced plasticity.

\section{HOW DOES rTMS INDUCE NEURAL PLASTICITY?}

During the past years experimental evidence has emerged which suggests that rTMS-induced after-effects are mediated by "longterm potentiation (LTP)-like" mechanisms (Ziemann et al., 2008). This "evidence" is based on physiological characteristics and pharmacological analogies between studies performed at the system level in human subjects and data obtained from animal models using classic LTP-experiments (see, Hoogendam et al., 2010). Yet, it has not been conclusively demonstrated at the single cell level that magnetic stimulation increases excitatory synaptic strength of cortical neurons. Using organotypic entorhino-hippocampal slice cultures we were recently able to provide experimental evidence that rMS can induce functional and structural changes of CA1 pyramidal neurons (Vlachos etal., 2012), which are consistent with a LTP of AMPA-R mediated synaptic transmission as seen after local electrical stimulation (Malenka and Bear, 2004). In contrast to classic LTP-protocols, however, in our study NMDA-R-mediated strengthening of excitatory postsynapses and an enlargement of dendritic spines were induced by a $10 \mathrm{~Hz}$ rMS-protocol, i.e., at comparatively low stimulation frequencies (Vlachos et al., 2012). Given the considerations above on TMS-effects during stimulation (Figure 2A) one may hypothesize that the induction of LTP at low stimulation frequencies is explained by the highly efficient recruitment of Hebbian-type plasticity mechanisms (Hebb, 1949) via the simultaneous (and repeated) activation of pre- and postsynaptic structures during rMS. Indeed, compartmental modeling suggests that a cooperative effect of bAP-induced dendritic depolarization and aAP-induced synaptic transmission seems sufficient to explain some of the observed rMS-induced effects on excitatory synapses of cultured CA1 pyramidal neurons (unpublished work). However, direct evidence for this "bAP-aAP theory" is currently missing. It also remains to be shown whether the precise timing of bAP-induced dendritic depolarization and aAP-mediated synaptic transmission (which will be delayed at neurochemical synapses) can lead to differential effects along the dendritic tree (for a recent review on spike timing dependent plasticity see, Feldman, 2012). Moreover, rMS-induced plasticity of inhibitory synapses and intrinsic cellular properties need to be assessed in this context (Figure 2B).

Apparently, these considerations do not take the specific fiber and cytoarchitecture of the stimulated network into account. It will therefore be important to compare the effects of a given $\mathrm{r}(\mathrm{T}) \mathrm{MS}$-protocol in different brain regions (e.g., hippocampal region CA1 vs. dentate gyrus; ideally different neocortical regions). These studies can provide new important insights on the role of local circuitries in rTMS-induced plasticity. As discussed by Funke and Benali (2011), however, a major limitation of performing these experiments in the intact animal is the comparatively small brain size. The smallest coils available are still too large to selectively stimulate specific cortical regions of mice or other small animals.

\section{ARE OTHER FORMS OF PLASTICITY INDUCED BY rTMS}

Several studies in human subjects have demonstrated rTMS-effects on cortical plasticity in the absence of detectable after-effects on cortical excitability (e.g., Hamada et al., 2008; Wankerl et al., 2010; Murakami et al., 2012). Hence, it appears important to consider rTMS-effects, which modulate the ability of neurons to express synaptic plasticity without (or beyond) changing the excitability of the stimulated network. The term metaplasticity describes this ability of neurons to change their state which influences the direction, magnitude and duration of future synaptic changes (for a recent review see, Hulme et al., 2013). It has been proposed that such "state-dependency" of synaptic plasticity could explain part of the inter- and intra-individual variability of rTMS-induced after-effects (Ridding and Ziemann, 2010). Conversely, it has been suggested that rTMS can "prime," i.e., change the plasticity state of neuronal networks (Ziemann and Siebner, 2008). Thus, rTMS may be used as a therapeutic tool to modulate, or even restore the ability of neurons to express synaptic plasticity under pathological conditions.

A straightforward approach to test for rMS-induced metaplasticity at the cellular level is combining rMS-experiments with classic LTP- or long-term depression (LTD)-protocols (i.e., local high- or low-frequency electrical stimulation). Our work in organotypic slice cultures (Vlachos et al., 2012) has shown that the rMSinduced increase in excitatory synaptic strength of CA1 pyramidal neurons returns back to baseline $6-8 \mathrm{~h}$ following stimulation. It will now be interesting to test for metaplasticity by comparing the ability to induce LTP and/or LTD in cultures 6-8 h after magnetic stimulation with LTP/LTD-induction in age-and timematched non-stimulated control cultures. A systematic evaluation of rMS-induced metaplasticity should include the assessment of (i) different intervals between rMS and classic LTP/LTD-induction, (ii) the effects of different rMS-protocols (including multiple sessions of rMS), and (iii) the molecular mechanisms of rMS-induced metaplasticity (e.g., by using pharmacological and/or genetic approaches). Likewise, rTMS-effects on synaptic tagging and capture (Redondo and Morris, 2011) and homeostatic synaptic plasticity (Turrigiano, 2012; Vitureira et al., 2012; Davis, 2013) should be evaluated. A better understanding of rMS-induced metaplasticity (and other non-Hebbian forms of plasticity) may thus help to devise rTMS-based priming strategies for optimized learning by physical exercise, language, or cognitive training in clinical neuro-restoration.

\section{ARE STRUCTURAL CHANGES OF NEURONS INDUCED BY rTMS?}

The induction of structural plasticity, i.e., the activity-dependent re-wiring of neuronal networks is another interesting mechanism by which rTMS could assert long-lasting effects on neuronal circuits. Changes in dendritic spine/synapse numbers, dendritic remodeling, and/or axonal sprouting can all affect connectivity and thus function of neuronal networks. During the past decade advances in live-cell imaging and in vivo microscopy have made it possible to monitor structural plasticity for long periods of time (weeks and months) at high resolution both in vitro and in vivo (Holtmaat et al., 2009). It has become possible to monitor structures down to the level 
of single molecules using modern diffraction-breaking imaging techniques (for recent review see, Tonnesen and Nagerl, 2013). These new techniques open up a window to rMS-induced changes at the subcellular (e.g., mitochondria, intracellular calcium stores) and molecular level (e.g., postsynaptic scaffolds; Figure 2B; see also Choquet and Triller, 2013). Since live-cell imaging techniques have only been used in a single rMS-study so far (Vlachos etal., 2012), we expect a wealth of important new data on rMS-induced structural plasticity during the next years.

\section{HOW CAN KNOWLEDGE ON THE CELLULAR AND MOLECULAR MECHANISMS OF rTMS BE TRANSLATED INTO CLINICAL PRACTICE?}

A major advantage of investigating the cellular and molecular mechanisms of rMS using appropriate animal models or in vitro preparations (e.g., of the hippocampus or neocortex) is the fact that various forms of plasticity (LTP/LTD, STDP of excitatory and inhibitory synapses, intrinsic cellular plasticity, metaplasticity, structural plasticity) have been studied in great detail using these models during the past decades. Data on rMS-induced neural plasticity can therefore be compared with results obtained in these in vivo or in vitro studies, which will facilitate the identification of the molecular mechanisms underlying rTMS-induced plasticity.

Since animal models of a large variety of brain diseases exist, it will be important to also study mechanisms of rTMS-induced neural plasticity in these models. Together with experiments performed under physiological conditions this knowledge will be helpful in addressing some of the current limitations/questions regarding the therapeutic use of rTMS. For example, it is not clear how rTMS-induced Hebbian plasticity, i.e., an increase in cortical excitability independent of a specific task, links to behavioral effects and could thus modulate the course of a neurologi$\mathrm{cal} /$ neuropsychiatric disease. Accordingly, future studies in animal models need to address the question of whether it is rTMS-induced Hebbian plasticity and/or the induction of metaplasticity and/or interference with other plasticity-forms such as structural plasticity [or denervation-induced homeostatic plasticity (Vlachos et al., 2013)], which underlies the therapeutic effects of rTMS. These studies will also be helpful to confirm (or re-interpret) results obtained in human studies in which rTMS has been applied in conjunction with pharmacological treatments (e.g., Maarrawi et al., 2007; Fonoff et al., 2009; de Andrade et al., 2011; for review see Nitsche et al., 2012).

Eventually the knowledge gained from animal studies may be translated into clinical practice (i) by optimizing the efficacy and specificity of the stimulation to induce and/or modulate certain forms of neural plasticity with rTMS; (ii) by using knowledge about the state-dependency of rTMS-induced plasticity (e.g., by understanding the role of genetic polymorphisms, neuromodulators, metaplasticity, or homeostatic synaptic plasticity); or (iii) by combining rTMS with pharmacological interventions in order to support specific rTMS effects [an approach termed "pharmaco-TMS"; (Ziemann, 2011)]. A better knowledge of the cellular and molecular mechanisms of rMS will therefore help to optimize rTMS-based therapies and could be a step toward "personalized" rTMStreatments of patients with neurological or neuropsychiatric diseases.

\section{ACKNOWLEDGMENTS}

We thank Drs. T. Deller, P. Jedlicka, and U. Ziemann for helpful discussions.

\section{REFERENCES}

Akerboom, J., Carreras Calderón, N., Tian, L., Wabnig, S., Prigge, M., Tolö, J., et al. (2013). Genetically encoded calcium indicators for multi-color neural activity imaging and combination with optogenetics. Front. Mol. Neurosci. 6:2. doi: 10.3389/fnmol.2013.00002

Barker, A. T., Jalinous, R., and Freeston, I. L. (1985). Non-invasive magnetic stimulation of human motor cortex. Lancet 1, 1106-1107. doi: 10.1016/S01406736(85)92413-4

Basser, P. J. (1994). Focal magnetic stimulation of an axon. IEEE Trans. Biomed. Eng. 41, 601-606. doi: 10.1109/10.293248

Basser, P. J., and Roth, B. J. (1991). Stimulation of a myelinated nerve axon by electromagnetic induction. Med. Biol. Eng. Comput. 29, 261-268. doi: 10.1007/BF02446708

Benali, A., Trippe, J., Weiler, E., Mix, A., Petrasch-Parwez, E., Girzalsky, W., et al. (2011). Theta-burst transcranial magnetic stimulation alters cortical inhibition. J. Neurosci. 31, 1193-1203. doi: 10.1523/JNEUROSCI.1379-10.2011

Berglund, K., Kuner, T., Feng, G., and Augustine, G. J. (2011). Imaging synaptic inhibition with the genetically encoded chloride indicator Clomeleon. Cold Spring Harb. Protoc. 2011, 1492-1497. doi: 10.1101/pdb.prot066985

Bhargava, Y., Hampden-Smith, K., Chachlaki, K., Wood, K. C., Vernon, J., Allerston, C. K., et al. (2013). Improved genetically-encoded, FlincG-type fluorescent biosensors for neural cGMP imaging. Front. Mol. Neurosci. 6:26. doi: $10.3389 /$ fnmol.2013.00026

Bliss, T. V., and Lomo, T. (1973). Long-lasting potentiation of synaptic transmission in the dentate area of the anaesthetized rabbit following stimulation of the perforant path. J. Physiol. 232, 331-356.

Bosch, M., and Hayashi, Y. (2012). Structural plasticity of dendritic spines. Curr. Opin. Neurobiol. 22, 383-388. doi: 10.1016/j.conb.2011.09.002

Bregestovski, P., Waseem, T., and Mukhtarov, M. (2009). Genetically encoded optical sensors for monitoring of intracellular chloride and chloride-selective channel activity. Front. Mol. Neurosci. 2:15. doi: 10.3389/neuro.02.015.2009

Chen, T. W., Wardill, T. J., Sun, Y., Pulver, S. R., Renninger, S. L., Baohan, A., et al. (2013). Ultrasensitive fluorescent proteins for imaging neuronal activity. Nature 499, 295-300. doi: 10.1038/nature12354

Choquet, D., and Triller, A. (2013). The dynamic synapse. Neuron 80, 691-703. doi: 10.1016/j.neuron.2013.10.013

Chipchase, L., Schabrun, S., Cohen, L., Hodges, P., Ridding, M., Rothwell, J., etal. (2012). A checklist for assessing the methodological quality of studies using transcranial magnetic stimulation to study the motor system: an international consensus study. Clin. Neurophysiol. 123, 1698-1704. doi: 10.1016/j.clinph.2012.05.003

Colgan, L. A., and Yasuda, R. (2013). Plasticity of dendritic spines: subcompartmentalization of signaling. Annu. Rev. Physiol. 76, 16.2-16.21. doi: 10.1146/annurev-physiol-021113-170400

Davis, G. W. (2013). Homeostatic signaling and the stabilization of neural function. Neuron 80, 718-728. doi: 10.1016/j.neuron.2013.09.044

de Andrade, D. C., Mhalla, A., Adam, F., Texeira, M. J., and Bouhassira, D. (2011). Neuropharmacological basis of rTMS-induced analgesia: the role of endogenous opioids. Pain 152, 320-326. doi: 10.1016/j.pain.2010.10.032

Deisseroth, K., and Schnitzer, M. J. (2013). Engineering approaches to illuminating brain structure and dynamics. Neuron 80, 568-577. doi: 10.1016/j.neuron.2013.10.032

Edgley, S. A., Eyre, J. A., Lemon, R. N., and Miller, S. (1997). Comparison of activation of corticospinal neurons and spinal motor neurons by magnetic and electrical transcranial stimulation in the lumbosacral cord of the anaesthetized monkey. Brain 120, 839-853. doi: 10.1093/brain/120.5.839

Edwards, M. J., Talelli, P., and Rothwell, J. C. (2008). Clinical applications of transcranial magnetic stimulation in patients with movement disorders. Lancet Neurol. 7, 827-840. doi: 10.1016/S1474-4422(08)70190-X 
Feldman, D. E. (2012). The spike-timing dependence of plasticity. Neuron 75, 556 571. doi: 10.1016/j.neuron.2012.08.001

Fifková, E., and Anderson, C. L. (1981). Stimulation-induced changes in dimensions of stalks of dendritic spines in the dentate molecular layer. Exp. Neurol. 74, 621-627. doi: 10.1016/0014-4886(81)90197-7

Fifková, E., and Van Harreveld, A. (1977). Long-lasting morphological changes in dendritic spines of dentate granular cells following stimulation of the entorhina area. J. Neurocytol. 6, 211-230. doi: 10.1007/BF01261506

Fonoff, E. T., Dale, C. S., Pagano, R. L., Paccola, C. C., Ballester, G., Teixeira, M. J., et al. (2009). Antinociception induced by epidural motor cortex stimulation in naive conscious rats is mediated by the opioid system. Behav. Brain Res. 196, 63-70. doi: 10.1016/j.bbr.2008.07.027

Funke, K., and Benali, A. (2011). Modulation of cortical inhibition by rTMS findings obtained from animal models. J. Physiol. 589, 4423-4434. doi: 10.1113/jphysiol.2011.206573

Galvani, A. (1791). De viribus electricitatis in motu musculari commentaris. Bononien Sci. Art Instit. Acad. Comm. 7, 363-418.

George, M. S., Taylor, J. J., and Short, E. B. (2013). The expanding evidence base for rTMS treatment of depression. Curr. Opin. Psychiatry 26, 13-18. doi 10.1097/YCO.0b013e32835ab46d

Gersner, R., Kravetz, E., Feil, J., Pell, G., and Zangen, A. (2011). Long-term effects of repetitive transcranial magnetic stimulation on markers for neuroplasticity: differential outcomes in anesthetized and awake animals. J. Neurosci. 31, 75217526. doi: 10.1523/JNEUROSCI.6751-10.2011

Gray, E. G. (1959). Axo-somatic and axo-dendritic synapses of the cerebral cortex: an electron microscope study. J. Anat. 93, 420-433.

Grewe, B. F., Langer, D., Kasper, H., Kampa, B. M., and Helmchen, F. (2010). High-speed in vivo calcium imaging reveals neuronal network activity with nearmillisecond precision. Nat. Methods 7, 399-405. doi: 10.1038/nmeth.1453

Grienberger, C., and Konnerth, A. (2012). Imaging calcium in neurons. Neuron 73 862-885. doi: 10.1016/j.neuron.2012.02.011

Grinvald, A., and Hildesheim, R. (2004). VSDI: a new era in functional imaging of cortical dynamics. Nat. Rev. Neurosci. 5, 874-885. doi: 10.1038/nrn1536

Hallett, M. (2007). Transcranial magnetic stimulation: a primer. Neuron 55, $187-$ 199. doi: 10.1016/j.neuron.2007.06.026

Hamada, M., Murase, N., Hasan, A., Balaratnam, M., and Rothwell, J. C. (2013). The role of interneuron networks in driving human motor cortical plasticity. Cereb Cortex 23, 1593-1605. doi: 10.1093/cercor/bhs147

Hamada, M., Terao, Y., Hanajima, R., Shirota, Y., Nakatani-Enomoto, S. Furubayashi, T., et al. (2008). Bidirectional long-term motor cortical plasticity and metaplasticity induced by quadripulse transcranial magnetic stimulation. $J$ Physiol. 586, 3927-3947. doi: 10.1113/jphysiol.2008.152793

Hebb, D. O. (1949). The Organization of Behavior: a Neuropsychological Theory. New York: Wiley.

Hoogendam, J. M., Ramakers, G. M., and Di Lazzarro, V. (2010). Physiology of repetitive transcranial magnetic stimulation of the human brain. Brain Stimul. 3, 95-118. doi: 10.1016/j.brs.2009.10.005

Holtmaat, A., Bonhoeffer, T., Chow, D. K., Chuckowree, J., De Paola, V., Hofer, S. B., et al. (2009). Long-term, high-resolution imaging in the mouse neocortex through a chronic cranial window. Nat. Protoc. 4, 1128-1144. doi 10.1038/nprot.2009.89

Hulme, S. R., Jones, O. D., and Abraham, W. C. (2013). Emerging roles of metaplasticity in behaviour and disease. Trends Neurosci. 36, 353-362. doi: 10.1016/j.tins.2013.03.007

Levkovitz, Y., Marx, J., Grisaru, N., and Segal, M. (1999). Long-term effects of transcranial magnetic stimulation on hippocampal reactivity to afferent stimulation. J. Neurosci. 19, 3198-3203.

Maarrawi, J., Peyron, R., Mertens, P., Costes, N., Magnin, M., Sindou, M., et al. (2007). Motor cortex stimulation for pain control induces changes in the endogenous opioid system. Neurology 69, 827-834. doi: 10.1212/01.wnl.0000269783.86997.37

Malenka, R. C., and Bear, M. F. (2004). LTP and LTD: an embarrassment of riches. Neuron 44, 5-21. doi: 10.1016/j.neuron.2004.09.012

Marshel, J. H., and Deisseroth, K. (2013). Genetically encoded voltage sensor goes live. Nat. Biotechnol. 31, 994-995. doi: 10.1038/nbt.2738

Meyer, J. F., Wolf, B., and Gross, G. W. (2009). Magnetic stimulation and depression of mammalian networks in primary neuronal cell cultures. IEEE Trans. Biomed. Eng. 56, 1512-1523. doi: 10.1109/TBME.2009.2013961
Mix, A., Benali, A., and Funke, K. (2013). Strain differences in the effect of rTMS on cortical expression of calcium-binding proteins in rats. Exp. Brain Res. doi: 10.1007/s00221-013-3751-3756 [Epub ahead of print].

Müller-Dahlhaus, J. F., Orekhov, Y., Liu, Y., and Ziemann, U. (2008). Interindividual variability and age-dependency of motor cortical plasticity induced by paired associative stimulation. Exp. Brain Res. 187, 467-475. doi: 10.1007/s00221-0081319-7

Murakami, T., Müller-Dahlhaus, F., Lu, M. K., and Ziemann, U. (2012). Homeostatic metaplasticity of corticospinal excitatory and intracortical inhibitory neural circuits in human motor cortex. J. Physiol. 590, 5765-5781. doi: 10.1113/jphysiol.2012.238519

Nitsche, M. A., Müller-Dahlhaus, F., Paulus, W., and Ziemann, U. (2012). The pharmacology of neuroplasticity induced by non-invasive brain stimulation: building models for the clinical use of CNS active drugs. J. Physiol. 590, 4641-4662. doi: 10.1113/jphysiol.2012.232975

Opitz, A., Windhoff, M., Heidemann, R. M., Turner, R., and Thielscher, A. (2011). How the brain tissue shapes the electric field induced by transcranial magnetic stimulation. Neuroimage 58, 849-859. doi: 10.1016/j.neuroimage.2011.06.069

Packer, A. M., Roska, B., and Hausser, M. (2013). Targeting neurons and photons for optogenetics. Nat. Neurosci. 16, 805-815. doi: 10.1038/nn.3427

Perron, A., Mutoh, H., Akemann, W., Gautam, S. G., Dimitrov, D., Iwamoto, Y., et al. (2009). Second and third generation voltage-sensitive fluorescent proteins for monitoring membrane potential. Front. Mol. Neurosci. 2:5. doi: 10.3389/neuro.02.005.2009

Raimondo, J. V., Irkle, A., Wefelmeyer, W., Newey, S. E., and Akerman, C. J. (2012). Genetically encoded proton sensors reveal activity-dependent $\mathrm{pH}$ changes in neurons. Front. Mol. Neurosci. 5:68. doi: 10.3389/fnmol.2012.00068

Redondo, R. L., and Morris, R. G. (2011). Making memories last: the synaptic tagging and capture hypothesis. Nat. Rev. Neurosci. 12, 17-30. doi: 10.1038/nrn2963

Ridding, M. C., and Ziemann, U. (2010). Determinants of the induction of cortical plasticity by non-invasive brain stimulation in healthy subjects. J. Physiol. 588, 2291-2304. doi: 10.1113/jphysiol.2010.190314

Rotem, A., and Moses, E. (2008). Magnetic stimulation of one-dimensional neuronal cultures. Biophys. J. 94, 5065-5078. doi: 10.1529/biophysj.107.125708

Scanziani, M., and Hausser, M. (2009). Electrophysiology in the age of light. Nature 461, 930-939. doi: 10.1038/nature08540

Smedemark-Margulies, N., and Trapani, J. G. (2013). Tools, methods, and applications for optophysiology in neuroscience. Front. Mol. Neurosci. 6:18. doi: 10.3389/fnmol.2013.00018

Spacek, J. (1985). Three-dimensional analysis of dendritic spines. II. Spine apparatus and other cytoplasmic components. Anat. Embryol. (Berl.) 171, 235-243. doi: 10.1007/BF00341418

Stuart, G. J., and Sakmann, B. (1994). Active propagation of somatic action potentials into neocortical pyramidal cell dendrites. Nature 367, 69-72. doi: $10.1038 / 367069 \mathrm{a} 0$

Tecchio, F., Zappasodi, F., Pasqualetti, P., De Gennaro, L., Pellicciari, M. C., Ercolani, M., et al. (2008). Age dependence of primary motor cortex plasticity induced by paired associative stimulation. Clin. Neurophysiol. 119, 675-682. doi: 10.1016/j.clinph.2007.10.023

Tokay, T., Holl, N., Kirschstein, T., Zschorlich, V., and Kohling, R. (2009). High-frequency magnetic stimulation induces long-term potentiation in rat hippocampal slices. Neurosci. Lett. 461, 150-154. doi: 10.1016/j.neulet.2009. 06.032

Tonnesen, J., and Nagerl, U. V. (2013). Superresolution imaging for neuroscience. Exp. Neurol. 242, 33-40. doi: 10.1016/j.expneurol.2012.10.004

Turrigiano, G. (2012). Homeostatic synaptic plasticity: local and global mechanisms for stabilizing neuronal function. Cold Spring Harb. Perspect. Biol. 4, a005736. doi: $10.1101 /$ cshperspect.a005736

Tye, K. M., and Deisseroth, K. (2012). Optogenetic investigation of neural circuits underlying brain disease in animal models. Nat. Rev. Neurosci. 13, 251-266. doi: $10.1038 / \mathrm{nrn} 3171$

Van Harreveld, A., and Fifková, E. (1975). Swelling of dendritic spines in the fascia dentata after stimulation of the perforant fibers as a mechanism of posttetanic potentiation. Exp. Neurol. 49, 736-749. doi: 10.1016/0014-4886(75) 90055-2

Vitureira, N., Letellier, M., and Goda, Y. (2012). Homeostatic synaptic plasticity: from single synapses to neural circuits. Curr. Opin. Neurobiol. 22, 516-521. doi: 10.1016/j.conb.2011.09.006 
Vlachos, A., Müller-Dahlhaus, F., Rosskopp, J., Lenz, M., Ziemann, U., and Deller, T. (2012). Repetitive magnetic stimulation induces functional and structural plasticity of excitatory postsynapses in mouse organotypic hippocampal slice cultures. J. Neurosci. 32, 17514-17523. doi: 10.1523/JNEUROSCI.040912.2012

Vlachos, A., Helias, M., Becker, D., Diesmann, M., and Deller, T. (2013). NMDAreceptor inhibition increases spine stability of denervated mouse dentate granule cells and accelerates spine density recovery following entorhinal denervation in vitro. Neurobiol. Dis. 59, 267-276. doi: 10.1016/j.nbd.2013.07.018

Wang, H. Y., Crupi, D., Liu, J., Stucky, A., Cruciata, G., Di Rocco, A., et al. (2011). Repetitive transcranial magnetic stimulation enhances BDNF-TrkB signaling in both brain and lymphocyte. J. Neurosci. 31, 11044-11054. doi: 10.1523/JNEUROSCI.2125-11.2011

Wankerl, K., Weise, D., Gentner, R., Rumpf, J. J., and Classen, J. (2010) L-type voltage-gated $\mathrm{Ca} 2+$ channels: a single molecular switch for long-term potentiation/long-term depression-like plasticity and activity-dependent metaplasticity in humans. J. Neurosci. 30, 6197-6204. doi: 10.1523/JNEUROSCI.467309.2010

Ziemann, U. (2005). Improving disability in stroke with RTMS. Lancet Neurol. 4 454-455. doi: 10.1016/S1474-4422(05)70126-5

Ziemann, U. (2011). Transcranial magnetic stimulation at the interface with other techniques: a powerful tool for studying the human cortex. Neuroscientist 17, 368-381. doi: 10.1177/1073858410390225
Ziemann, U., Paulus, W., Nitsche, M. A., Pascual-Leone, A., Byblow, W. D., Berardelli, A., et al. (2008). Consensus: motor cortex plasticity protocols. Brain Stimul. 1, 164-182. doi: 10.1016/j.brs.2008.06.006

Ziemann, U., and Siebner, H. R. (2008). Modifying motor learning through gating and homeostatic metaplasticity. Brain Stimul. 1, 60-66. doi: 10.1016/j.brs.2007.08.003

Conflict of Interest Statement: The authors declare that the research was conducted in the absence of any commercial or financial relationships that could be construed as a potential conflict of interest.

Received: 29 September 2013; accepted: 29 November 2013; published online: 17 December 2013.

Citation: Müller-Dahlhaus F and Vlachos A (2013) Unraveling the cellular and molecular mechanisms of repetitive magnetic stimulation. Front. Mol. Neurosci. 6:50. doi: 10.3389/fnmol.2013.00050

This article was submitted to the journal Frontiers in Molecular Neuroscience.

Copyright (C) 2013 Müller-Dahlhaus and Vlachos. This is an open-access article distributed under the terms of the Creative Commons Attribution License (CC BY). The use, distribution or reproduction in other forums is permitted, provided the original author(s) or licensor are credited and that the original publication in this journal is cited, in accordance with accepted academic practice. No use, distribution or reproduction is permitted which does not comply with these terms. 